\title{
Pancreatic surgery, not pancreatitis, is the primary cause of diabetes after acute fulminant pancreatitis
}

\author{
J Eriksson, M Doepel, E Widén, L Halme, A Ekstrand, L Groop, K Höckerstedt
}

\begin{abstract}
Acute fulminant pancreatitis is associated with significant morbidity and mortality. To examine the outcome of conservative and surgical treatment of this disorder, 36 patients who survived an initial episode were restudied after a mean of six years. Fifty three per cent had developed diabetes mellitus, half of whom required insulin therapy. Pancreatic resection was associated with a $100 \%$ frequency of diabetes, while only $26 \%$ of those treated with peritoneal lavage developed this $(p<0.001)$. Insulin secretion and sensitivity were assessed using the hyperglycaemic glucose clamp technique. First phase insulin secretion was impaired in surgically treated patients (mean (SEM) 14 (5) $\mu \mathrm{U} / \mathrm{ml} \times 10$ minutes) compared with conservatively treated patients and control subjects (144 (66) and $87(12) \mu \mathrm{U} / \mathrm{ml} \times 10$ minutes, respectively; $\mathbf{p}<0.05)$. Second phase and 'maximal' insulin secretion were also impaired among the surgically treated patients compared with the conservatively treated patients and the controls. Insulin sensitivity was reduced among the surgically treated patients $(2.88(58) \mathrm{mg} / \mathrm{kg}$.minute) when compared with conservatively treated patients and healthy control subjects $(5.87(1.02)$ and 6.45 $(0.66) \mathrm{mg} / \mathrm{kg}$.minute; $\mathrm{p}<0.05)$. Pancreatic resection is associated with a very high frequency of diabetes compared with peritoneal lavage, and these results favour conservative treatment of active fulminant pancreatitis whenever possible.
\end{abstract}

Acute fulminant pancreatitis represents about $5-15 \%$ of all cases of acute pancreatitis. Apart from a high mortality rate of $13-64 \%$, it is associated with a high frequency of secondary diabetes. Although it is generally agreed that pancreatic $\beta$-cell loss as a result of the pancreatic disease is the cause of diabetes, it is not known whether other factors such as the mode of treatment contribute to the development of diabetes after active fulminant pancreatitis. The two most common invasive methods used in the treatment of active fulminant pancreatitis are pancreatic resection and peritoneal lavage. These have been considered superior to conservative treatment alone. . $^{1-10}$

The present study aimed to examine the outcome of surgical and conservative therapy in active fulminant pancreatitis and their influence on insulin secretion and glucose metabolism.

\section{Methods and subjects}

From 1974 to 1988,67 patients with active fulminant pancreatitis were treated at the IV Department of Surgery at Helsinki University
Central Hospital. The primary mortality rate was $34 \%(n=23)$, and four of the survivors died after hospital discharge during the follow up period. As four more patients could not be reached, the final follow up was performed in 36 subjects - that is, $90 \%$ of the survivors. Of the 36 patients, 24 were men and 12 women. The mean age at onset of active fulminant pancreatitis for the whole study group was 43 years (range 23-86 years).

The aetiology of the pancreatic disease was alcohol in $78 \%$, biliary tract disease in $6 \%$, and endoscopic retrograde cholangiopancreatography in $6 \%$; in $11 \%$ the active fulminant pancreatitis was caused by unknown factors. Hypertriglyceridaemia, hyperparathyroidism, and corticosteroid treatment were all excluded as aetiological factors. The findings in the acute phase for most of the patients are reported in reference 6.

The term acute fulminant pancreatitis is used for the multiple organ failure disease which is seen in patients with necrotic or haemorrhagic pancreatitis, or both. The diagnosis was based upon clinical tests and computed tomography findings. In 33 of the 36 patients a diagnostic laparatomy was performed and the pancreas was found to be haemorrhagic or necrotic in all cases. Serum amylase activities were raised in all patients. The primary treatment in all included peritoneal lavage and parenteral hyperalimenation in the intensive care unit. The pancreatic region was rinsed every hour with 1 litre of a standard peritoneal lavage solution, 24 hours daily, for a mean of 10 days. If, during the primary treatment period, a second sudden deterioration occurred or new organ failures developed, the treatment was regarded as insufficient and pancreatic resection was performed. All patients were treated by one of the authors $(\mathrm{KH})$.

There was no difference in the severity of the acute fulminant pancreatitis at admission to hospital between those patients who underwent resection compared with those treated conservatively. According to the Ranson criteria, $60 \%$ of the patients in the resection group compared with $53 \%$ in the lavage group had a severe pancreatitis."

The 36 patients underwent an oral glucose tolerance test at a mean of $6 \cdot 2$ years (range $1-14$ years) after the episode of active fulminant pancreatitis. During follow up eight subjects (22\%) experienced at least one further attack of acute pancreatitis. All subjects were asked to participate in another study to evaluate insulin secretion and insulin sensitivity by a hyperglycaemic clamp technique. Twenty three patients responded to the request but eight refused to participate (four because of continued alcohol abuse, two for 
psychological reasons, and two for other personal reasons). Two patients lived abroad and could not be reached and three patients were over 85 years of age. All these subjects were excluded from the hyperglycaemic clamp study. In addition, nine of the remaining 23 patients had been put on insulin therapy because of insulin deficiency (verified by undetectable Cpeptide values), and did not undergo the hyperglycaemic clamp study. Of these nine patients, seven had had pancreatic resection. Therefore 14 patients participated in the follow up study for evaluation of insulin secretion and insulin sensitivity. Seven age and weight matched subjects with normal glucose tolerance and no family history of diabetes served as controls in the hyperglycaemic clamp studies.

\section{ORAL GLUCOSE TOLERANCE TEST AND}

BIOCHEMICAL TESTS

A two-hour oral glucose tolerance test was used to assess glucose tolerance in all subjects without diagnosed diabetes. After a 12 hour overnight fast, the subjects were given a $75 \mathrm{~g}$ oral glucose load. According to the World Health Organisation criteria, the subjects were divided into three groups - that is, those with normal, those with impaired glucose tolerance, and diabetic subjects. ${ }^{12}$

Biochemical tests including haemoglobin and leukocyte count, amylase, alanine aminotransferase, $\gamma$ glutamyltransferase, cholesterol, high density lipoprotein cholesterol, triglycerides, and glycohaemoglobin $A_{l c}$ were assessed.

\section{HYPERGLYCAEMIC GLUCOSE CLAMP}

Insulin secretion was evaluated with the hyperglycaemic glucose clamp technique. ${ }^{13}$ During the hyperglycaemic clamp the plasma glucose concentration was raised rapidly by $7 \mathrm{mmol} / \mathrm{l}$ above the baseline value with a priming infusion of $20 \%$ glucose. The desired hyperglycaemic value was maintained for 120 minutes by adjustment of the glucose infusion. At a constant plasma glucose concentration, the amount of glucose required to maintain hyperglycaemia equals the value for whole body glucose metabolism, provided there is no entry of glucose from the liver. The correction for urinary loss of glucose was made.

Under these conditions of constant hyperglycaemia, the normal plasma insulin response is biphasic. After 120 minutes of hyperglycaemia, $0.5 \mathrm{mg}$ of glucagon was administered intravenously in order to further stimulate the pancreatic $\beta$-cells. Samples for measurement of plasma glucose and serum insulin were drawn every second minute for the first 10 minutes. Thereafter, the glucose concentration was determined every five minutes and the insulin level every 10 minutes.

Indirect calorimetry was used at the baseline and during the last 60 minutes of the hyperglycaemic clamp, before glucagon administration, to estimate the net rates of carbohydrate and lipid oxidation. ${ }^{14}$ A computerised open circuit system was used to measure gas exchange through a transparent 251 polyvinyl chloride plastic canopy (Deltatrac, Datex, Helsinki, Finland). ${ }^{15}$ Protein oxidation was calculated from the urinary excretion of urea nitrogen before and during the clamp.

\section{ASSAYS AND CALCULATIONS}

Plasma glucose was assayed with a glucose oxidase method adapted for the Beckman Glucose Analyzer II (Beckman Instruments, Fullerton, Calif). The glycohaemoglobin concentration in blood was measured by high pressure liquid chromatography. The serum insulin concentration was measured by a double antibody radioimmunoassay (Pharmacia, Uppsala, Sweden).

First and second phase insulin secretory responses were estimated by calculating the incremental insulin area during the first 10 minutes and between 10 minutes and 120 minutes of the hyperglycaemic clamp. The 'maximal' insulin secretion - that is, the glucose and glucagon stimulated insulin release - was calculated from the incremental area between 120 and 150 minutes.

Net glucose oxidation and lipid oxidation rates were calculated from indirect calorimetric measurements at baseline and during the last 60 minutes of the clamp. Non-oxidative glucose metabolism (primarily the storage of glucose as glucogen) was calculated as the difference between the total body glucose metabolism and glucose oxidation. The constants used to calculate glucose, lipid, and protein oxidation from gas exchange data are available in reference 14.

\section{STATISTICAL ANALYSES}

All data are expressed as mean (SEM). All statistical analyses were performed with the use of a BMDP statistical package. ${ }^{16} \mathrm{~A}$ one way analysis of variance or the Welch test was used to test the equality of group means. Scheffe's method was used for multiple comparisons between group means. A p value less than 0.05 was considered statistically significant.

\section{Results}

TREATMENT AND DEVELOPMENT OF DIABETES

Thirteen patients $(36 \%)$ underwent pancreatic surgery because of exacerbation of the disease. Among these patients, six had a 50\% pancreatic resection, six had an $80 \%$ pancreatic resection, while one underwent late total pancreatectomy (two years later).

Nineteen patients $(53 \%)$ developed diabetes during the six year follow up, and four (11\%) patients showed impaired glucose tolerance. Six diabetics $(32 \%)$ were treated with diet alone, four $(21 \%)$ were taking oral hypoglycaemic agents and nine $(47 \%)$ were on insulin treatment (mean (SEM) insulin dose $=38$ (4) IU/day). Fifty eight per cent of the diabetics developed the disease during the primary hospital stay for active fulminant pancreatitis, $31 \%$ of them developed diabetes within the first five years after active fulminant pancreatitis, and $11 \%$ developed the 
disease later than five years after active fulminant pancreatitis.

All patients who underwent pancreatic resection developed diabetes, whereas the corresponding number for those treated conservatively was $26 \%(p=0 \cdot 0004)$. Sixty seven per cent of the men with active fulminant pancreatitis developed diabetes, while only $25 \%$ of the women developed the disease $(p<0 \cdot 05)$. The aetiology of active fulminant pancreatitis did not seem to influence the choice of treatment.

The only significant differences in the biochemical tests between those patients treated with resection and those treated with lavage were seen in the parameters related to the appearance of diabetes - that is, plasma glucose and glycohaemoglobin $A_{1 c}$ concentrations (Table).

\section{INSULIN SENSITIVITY AND INSULIN SECRETION}

Fourteen patients with a history of active fulminant pancreatitis and seven healthy control subjects participated in the hyperglycaemic clamp study. The patients were further divided into those treated with peritoneal lavage only $(n=9)$ and those treated surgically $(n=5)$. Two of these conservatively treated patients had developed diabetes while all those who had undergone surgery had developed diabetes.

Metabolic characteristics related to insulin sensitivity are given in the Table.

Insulin secretion during the clamp is shown graphically in the Figure. First phase insulin secretion (14 (5) $v 144$ (66), and 87 (12) $\mu \mathrm{U} / \mathrm{ml} \times$ 10 minutes; $\mathrm{p}<0.05)$ was impaired among the surgically treated group compared with the conservatively treated group and the controls. Second phase insulin secretion as well as 'maximal' insulin secretion were impaired in the surgically treated group compared with the conservatively treated subjects and controls. The decrease was not, however, statistically significant because of the small number of patients $(126$ (32) $v 280$ (139), and $175(47) \mu \mathrm{U} / \mathrm{ml} \times 110$ minutes; $\mathrm{p}=\mathrm{NS}$ ) and (243 (57), 514 (172), and $653(108) \mu \mathrm{U} / \mathrm{ml} \times 30$ minutes $)$.

\section{Discussion}

Diabetes secondary to pancreatopathy forms about $0.5 \%$ of all patients with diabetes. ${ }^{17}$ Diabetes can occur in all forms of pancreatic disease and total pancreatectomy is always accompanied by diabetes. It has been estimated

Metabolic characteristics of those subjects participating in the hyperglycaemic clamp studies

\begin{tabular}{|c|c|c|c|}
\hline & $\begin{array}{l}\text { Resection } \\
(n=5)\end{array}$ & $\begin{array}{l}\text { Lava- } \\
\text { tion } \\
(n=9)\end{array}$ & $\begin{array}{l}\text { Controls } \\
(n=7)\end{array}$ \\
\hline Fasting insulin $(\mu \mathrm{U} / \mathrm{ml})$ & $11(2)$ & $9(2)$ & $4(1)$ \\
\hline Fasting plasma glucose $(\mathrm{mmol} / \mathrm{l})$ & $8 \cdot 8(0 \cdot 8)^{\star \star}$ & $5 \cdot 7(0.3)$ & $5 \cdot 0(0 \cdot 2)$ \\
\hline Glycohaemoglobin $A_{1 \mathrm{c}}(\%)$ & $7 \cdot 6(0 \cdot 5)^{\star \star}$ & $5 \cdot 5(0 \cdot 2)$ & $5 \cdot 2(0 \cdot 2)$ \\
\hline Basal glucose oxidation (mg/kg.min) & $1.54(0.27)$ & $1.48(0 \cdot 16)$ & $1.43(0.09)$ \\
\hline Basal lipid oxidation (mg/kg.min) & $0.72(0.15)$ & $0.73(0.09)$ & $0.68(0.08)$ \\
\hline Insulin stimulated glucose disposal (mg/kg.min) & $2.88(0.58)^{\star}$ & $5.87(1.02)$ & $6.45(0.66)$ \\
\hline Insulin stimulated glucose oxidation (mg/kg.min) & $1.68(0.53)^{\star}$ & $2.65(0.30)$ & $2 \cdot 83(0 \cdot 16)$ \\
\hline \multirow{3}{*}{$\begin{array}{l}\text { Insulin stimulated non-oxidative glucose metabolism } \\
\text { (mg/kg.min) } \\
\text { Insulin suppressed lipid oxidation (mg/kg.min) }\end{array}$} & & & \\
\hline & $0.68(0.28)^{\star}$ & $3.34(0.99)$ & $3.14(0.55)$ \\
\hline & $0 \cdot 71(0 \cdot 18)$ & & $0 \cdot 36(0 \cdot 10)$ \\
\hline
\end{tabular}

${ }^{\star} \mathrm{p}<0.05 v$ lavation and controls; ${ }^{\star \star} \mathrm{p}<0.01 v$ lavation and controls.

Resection = acute fulminant pancreatitis patients treated with pancreas resection; lavation $=$ acute fulminant pancreatitis patients treated with peritoneal lavage only; controls=healthy control subjects
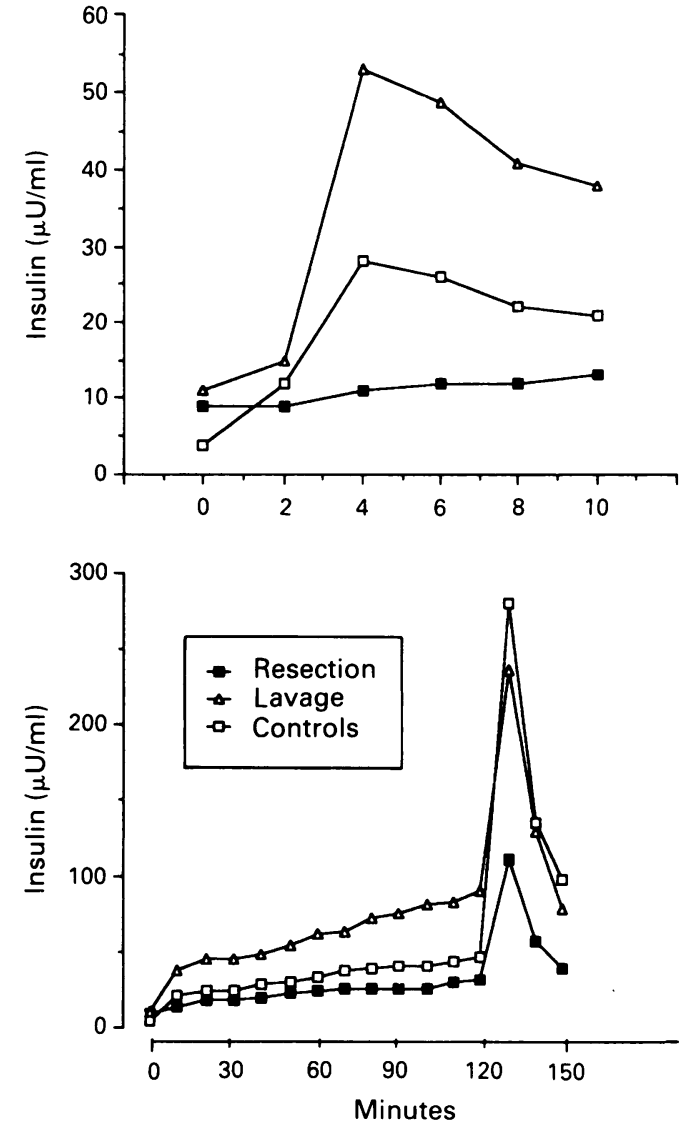

Mean serum insulin concentrations during the hyperglycaemic glucose clamp ( $+7 \mathrm{mmol} / \mathrm{l})$ in surgically treated patients, conservatively treated patients, and healthy control subjects. Glucagon $(0.5 \mathrm{mg})$ was given intravenously at 120 minutes. The first phase insulin responses (from 0 to 10 minutes) are shown enlarged.

that when 40 to $80 \%$ of the normal pancreas is removed there is a $32 \%$ risk of diabetes, while 80 to $95 \%$ removal is associated with a $72 \%$ risk of diabetes. ${ }^{18}$ Nordbeck et al observed that $92 \%$ of their patients developed diabetes during follow up after pancreatic resection for acute necrotising pancreatitis. ${ }^{19}$ This is in close agreement with the figures of the present study.

In chronic pancreatitis, reduced $\beta$-cell secretion is frequently observed leading to an increased risk of diabetes. The prevalence of diabetes in chronic pancreatitis has varied between 12 and $80 \% .^{18} 20$ The choice of treatment is of importance; Thow et al reported that $66 \%$ of their patients developed diabetes after surgical treatment compared with $21 \%$ after conservative treatment. $^{18}$ In contrast, the prevalence of diabetes after acute pancreatitis is rather low, ranging between 1 and $15 \% . .^{20-22}$ Only $26 \%$ of our conservatively treated patients with active fulminant pancreatitis developed diabetes after a follow up period of six years.

Diabetes after pancreatic surgery is characterised by insulin and glucagon deficiency and increased insulin sensitivity. ${ }^{23}$ 2t $\quad$ Diabetes secondary to pancreatic surgery differs therefore from the idiopathic forms of diabetes, which are usually characterised by insulin resistance. Glucagon deficiency accounts for some of the metabolic and clinical features, making diabetes secondary to pancreatic resection a well distinguished form of diabetes. Chronic glucagon deficiency reduces liver uptake of gluconeogenic 
precursors and results in low activity of gluconeogenic enzymes, leading to impaired capacity of the liver to produce glucose. The clinical counterpart of the lower rate of gluconeogenesis observed in 'pancreatogenic' diabetes is a lower fasting blood glucose concentration and increased insulin sensitivity. ${ }^{25}$ This means that patients with pancreatogenic diabetes require smaller doses of insulin than patients with idiopathic diabetes. ${ }^{26}$

Insulin sensitivity measured as glucose disposal during the hyperglycaemic glucose clamp study was severely impaired in the group of patients who had undergone pancreatic resection. The glucose disposal rate was more than $50 \%$ lower in the surgically treated patients than among the conservatively treated patients. The main reason for this decrease was impaired nonoxidative glucose metabolism - that is, mainly storage of glucose as glycogen in the muscle. A similar defect is characteristic for idiopathic type 2 diabetes. ${ }^{27}$ In keeping with the current results, in a previous study the rate of glucose metabolism was found to be $47 \%$ lower in diabetic patients with 'pancreatogenic' diabetes compared with normal subjects, using the euglycaemic insulin clamp technique. ${ }^{28}$

The normal insulin response to intravenous glucose is biphasic with a first phase response primarily representing formed and stored insulin and a second phase representing newly synthesised insulin..$^{14}$ A glucagon challenge two hours after the start of the glucose infusion results in insulin concentrations that are 10 fold higher than these observed during the second phase. It is assumed that the insulin concentrations are close to the maximal secretory capacity of the $\beta$ cells and thereby dependent upon the $\beta$-cell mass. In the patients developing diabetes, all phases of insulin secretion were impaired indicating that diabetes was the consequence of loss of $\beta$-cell mass. Since most of the patients who developed diabetes had undergone pancreatic resection, it is obvious that removal of pancreatic tissue is the major cause of diabetes. Interestingly, the patients treated conservatively showed normal insulin response and in particular a normal $\beta$-cell mass. These data indicate that patients treated conservatively with peritoneal lavage can survive active fulminant pancreatitis without any persistent defect in their $\beta$-cell function and strongly challenges the view that acute pancreatitis causes genuine and persistent $\beta$-cell damage. In this regard the results differ from chronic pancreatitis in which first phase insulin secretion frequently is impaired and the patients develop impaired glucose tolerance. ${ }^{29}$

However, insulin secretion in the group of patients with a history of active fulminant pancreatitis was heterogenous. Three different subgroups can be identified - those without endogenous insulin secretion, those with quantitatively impaired insulin secretion, and those with an apparently normal insulin secretion. The obvious resemblance to type 1 diabetes, type 2 diabetes, and healthy subjects cannot be left unmentioned.

Total duodenopancreatectomy has been questioned because of high figures of early and late mortality. Death as a result of severe hypo- glycaemia has been reported in $50 \%$ of patients undergoing total duodenopancreatectomy. Partial pancreatic resection or conservative treatment have been favoured, especially in patients with non-malignant disease. ${ }^{30}$ The treatment of acute fulminant pancreatitis has changed over the years and most centres today do not perform pancreatic resection in the way described some years ago. Debridement of necrotic tissue has become the accepted operative strategy. However, since these treatment methods have not been used for longer time periods, long term results are not available. The present study strongly supports conservative treatment in acute fulminant pancreatitis whenever possible as a means of reducing the risk of developing diabetes. It could of course be argued that only the more severe cases were operated on, but it seems certain that major pancreatic resection is associated with a high frequency of diabetes, while the prognosis with regard to diabetes, after conservative treatment is good. In resecting the body and tail of the pancreas - that is, the most $\beta$ cell rich part of the organ - the risk of diabetes increases considerably. ${ }^{31}$ It should also be kept in mind that secondary diabetes is associated with various complications caused by both hyperglycaemia and hypoglycaemia. These complications could also be avoided by more conservative treatment of acute fulminant pancreatitis.

1 Ranson JHC, Spencer FC. The tole of peritoneal lavage in severe acute pancreatitis. Ann Surg 1978; 187: 565-7.

2 Stone $\mathrm{HH}$, Fabian TC. Peritoneal dialysis in the treatment of acute pancreatitis. Surg Gynecol Obstet 1980; 150: 878-82.

3 Warshaw AL. A guide to pancreatitis. Compr Ther 1980; 6: 49-

4 Kivilaakso E, Fräki O, Nikki P, Lempinen M. Resection of the pancreas for acute fulminant pancreatitis. Surg Gyneco the pancreas for acute fulm
Obstet 1981;152: 492-8.

5 Ihse I, Evander A, Gustaf I. A controlled randomized study of the value of peritoneal lavage in acute pancreatitis. In: Hollender LF, ed. Controversies in acute pancreatitis. Berlin: Springer Verlag, 1982: 200-22.

6 Kauste A, Höckerstedt K, Ahonen J, Tervaskari H. Peritoneal lavage as the primary treatment in acute fulminant pancreatitis. Surg Gynecol Obstet 1983; 156: 458-63.

7 Kivilaakso E, Lempinen M, Mäkeläinen A, Nikki P, Schröder $T$. Pancreatic resection versus peritoneal lavation for acute fulminant pancreatitis. A randomized prospective study. Ann Surg 1984; 199: 426-31.

8 Lasson A, Balldin G, Genell S, Ohlsoon K. Peritoneal lavage in severe acute pancreatitis. Acta Chir Scand 1984; 150: 47984.

9 Mayer AD, McMahon MJ, Corfield AP, Fratton S, Brocco G, Marsoli $\mathrm{G}$, et al. Controlled clinical trial of peritoneal lavage for the treatment of severe acute pancreatitis. $N$ Engl $\mathcal{F}$ Med 1985; 312: 399-404.

10 Hollender LF, Meyer C, Keller D. Planned operations for necrotizing pancreatitis: the continuing experience. In Howard JM, Jordan GL, Reber HE, eds. Surgical diseases of the pancreas. Philadelphia: Lea and Febiger, 1986: 450-60.

11 Ranson JHC, Rifkind KM, Roses DF. Prognostic signs and the role of operative management in acute pancreatitis. Surg Gynecol Obstet 1974; 139: 69-81.

12 World Health Organisation. Diabetes mellitus. Technical report series. No 727. Geneva: WHO, 1985.

13 DeFronzo RA, Tobin JD, Andres R. Glucose clamp technique: a method for quantifying insulin secretion and nique: a method for quantifying insulin
resistance. Am $\mathcal{f}$ Physiol 1979; 237: E214-23.

14 Ferrannini E. The theoretical bases of indirect calorimetry: a review. Metabolism 1988; 37: 287-301.

15 Meriläinen PR. Metabolic monitor. Int $\mathcal{f}$ Clin Monit Comput 1987; 4: 167-77.

16 Dixon WJ, ed. BMDP statistical software manual. Berkeley, California: University of California Press, 1985.

17 Alberti KGMM. Diabetes secondary to pancreatopathy: an example of brittle diabetes. In: Tiengo A, Alberti KGMM DelPrato S, Vranic M, eds. Diabetes secondary to pan-
creatopathy. International Congress Series 762. Amsterdam: Excerpta Medica, 1988:211-4.

18 Thow J, Samad A, Alberti KGMM. Epidemiology and general aspects of diabetes secondary to pancreatopathy. In: Tiengo
A, Alberti KGMM, DelPrato S, Vranic M, eds. Diabetes secondary to pancreatopathy. International Congress Series secondary to pancreatopathy. International Cong.

19 Nordback IH, Auvinen OA. Long-term results after pancreas resection for acute necrotizing pancreatitis. Brf S Surg 1985; 72: 687-9. 
20 Scuro LA, Angelini G, Cavallini G, Vantini I. In: Gyr KL, Singer MV, Sarles H, eds. Pancreatitis - concepts and
classifications. International Congress Series 642. classifications. International Congress

21 Sinha SK. In: Podolsky S, Viswanathan M, eds. Secondary diabetes: the Spectrum of the diabetic syndrome. New York:

22 Trapnell JE. In: Podolsky S, Viswanathan M, eds. Secondary diabetes: the Spectrum of the diabetic syndrome. New York: Raven Press, 1980: 77-88.

23 Klujko J, Reiter J, Schwall G, Schmidt F. Glucagon is effective on glucose homeostasis in pancreatectomized diabetics. In: Tiengo A, Alberti KGMM, DelPrato S, Vranic $M$, eds. Diabetes secondary to pancreatopathy. International Congress Series 762. Amsterdam: Excerpta Medica, 1988: 169-77.

24 Baron AD, Schaefer L, Scragg P, Kolermann OG. Role of hyperglycagonemia in maintenance of increased rates of hepatic glucose output in Type II diabetes. Diabetes 1987; 36: $274-83$.

25 Nosadini R, DelPrato S, Tiengo A, Dummer E, Toffolo G, Cobelli $\mathrm{C}$, et al. Insulin sensitivity, binding, and kinetics in pancreatogenic and type I diabetes. Diabetes 1982;31: 346-53.

26 Bak M,Czyzyk A. Therapeutic problems in diabetes after tota pancreatectomy. In: 'Tiengo A, Alberti KGMM, DelPrato S, Vranic M, eds. Diabetes secondary to pancreatopathy. International Congress Series 762. Ämsterdam: Excerpta Medica: 1988: 239-43.

27 Shulman GI, Rothman DL, Jue T, Stein P, DeFronzo RA, Shulman RG. Quantitation of muscle glycogen synthesis in normal subjects and subjects with non-insulin-dependen diabetes by ${ }^{13} \mathrm{C}$ nuclear magnetic resonance spectroscopy. NEngl F Med 1990; 332: 223-8.

28 Yki-Järvinen H, Kiviluoto T, Taskinen Marja-Riitta. Insulin resistance is a prominent feature of patients with pancreatogenic diabetes. Metabolism 1986; 35: 718-27.

29 Joffe BJ, Bank S, Jackson WPU, Keller P, O'Reilly IG, Vinik AI. Insulin reserve in patients with chronic pancreatitis. Lancet 1968; ii: $890-2$.

30 Stanke AAR, Cuppers HJ, Berger M. Therapeutic problems in diabetes secondary to pancreatectomy. In: Tiengo A, Alberti KGMM, DePrato S, Vranic M, eds. Diabetes secondary to pancreatopathy. International Congress Series

31 Cook DL, Taborsky GJ Jr. B-cell function and insulin secretion. In: Rifkin $\mathrm{H}$, Porte D Jr, eds. Ellenberg and Rifkin's diabetes mellitus: theory and practice. New York Elsevier, 1990: 2241-5. 